\title{
Unterrichtsentwicklung in Zeiten der Systemreform
}

\section{Herbert Altrichter und Birgit Geisler}

Seit der ersten Hälfte der 1990 er Jahre sind in den verschiedenen deutschsprachigen Schulsystemen verstärkte Veränderungsbewegungen zu beobachten, die insgesamt als Versuche der Reform schulischer Steuerung interpretiert worden sind. Dabei rückt die Innovation des Unterrichts seit der zweiten Hälfte der 1990er Jahre verstärkt in den Fokus von Veränderungsforderungen. In diesem Sinne stellt sich der Beitrag die Frage, wie eine systemweite Reform im Mehrebenensystem Schule betrieben werden kann, die auch im Unterricht und in den Lernergebnissen der Schüler/innen spürbar wird. Dazu werden zunächst einige Erläuterungen zu unserem Verständnis von «neuer Steuerung» und dem Stellenwert von Unterricht darin gegeben (vgl. Kap. 1), um anschliessend die Auswirkung der gegenwärtig aktuellen Reforminstrumente "Bildungsstandards, standardbezogenen Tests und Datenfeedback» auf die Steuerung und Weiterentwicklung von Unterricht zu analysieren (vgl. Kap. 2). In Kapitel 3 werden schliesslich - in einer Umkehrung der Fragerichtung erfolgreiche Strategien der Unterrichtsentwicklung auf ihr Potenzial für systemweite Reformen befragt.

Nach einer auf bildungspolitischer Ebene «eigenartig unbewegte[n] Zeit» (Fend, 2006a, S. 225) in den 1980er Jahren erleben viele der deutschsprachigen Schulsysteme seit der ersten Hälfte der 1990er Jahre - und noch einmal verstärkt seit dem «PISA-Schock» 2001 - vielfältige Aktivitäten der Schulreform. Diese neue Phase der «Modernisierung der Schule» (Brüsemeister \& Eubel, 2003) hat sich in einer - für diese Bildungssysteme atemberaubend raschen - Folge von Reformen niedergeschlagen, in deren Zentrum einesteils zentral entworfene «neue Steuerungsinstrumente systemweiter Reform» (wie Bildungsstandards und standardbezogene Tests) stehen, die anderseits sich explizit zum Ziel gesetzt haben, gleichsam durch die verschiedenen Ebenen des Schulsystems hindurch den Unterricht zu verändern und damit das Schülerlernen und dessen Ergebnisse zu verbessern. Wie gehen diese beiden Interventionsideen - systemweite Steuerungsinstrumente und an der Basis spürbare Unterrichtsreform zusammen? Wie kann eine «systemweite» Reform im Mehrebenensystem 
«Schule» betrieben werden, die auch im Unterricht und in den Lernergebnissen der Schüler/innen spürbar wird? Diese Fragen sollen die folgenden Überlegungen leiten, an die wir aus zwei Richtungen herangehen wollen: Zunächst wollen wir fragen, ob «systemweite Entwicklungsstrategien», wie sie zentralen Stellenwert in den verschiedenen Varianten des neuen Steuerungsmodells aufweisen, Potenzial für Unterrichtsentwicklung haben? Dazu werden wir die Reforminstrumente «Bildungsstandards und Datenfeedback», die als der «unterrichtsfokussierte» Kern der aktuellen Versuche, eine «evidenzbasierte Steuerung und Entwicklung» des Schulsystems zu etablieren, erscheinen ${ }^{1}$, einer Analyse unterziehen. In einer zweiten Fragerichtung werden wir erfolgreiche Strategien der Unterrichtsentwicklung auf ihr Potenzial für systemweite Reformen befragen. Zuvor sollen jedoch in Kapitel 1 einige Erläuterungen zu unserem Verständnis von «neuer Steuerung» und dem Stellenwert von Unterricht darin gegeben werden.

\section{Unterrichtsreform und neues Steuerungsmodell}

Seit der ersten Hälfte der 1990er Jahre sind in den verschiedenen deutschsprachigen Schulsystemen verstärkte Veränderungsbewegungen zu beobachten, die insgesamt als Versuche der Reform schulischer Steuerung interpretiert worden sind. Trotz einiger Variationen in den deutschsprachigen Schulsystemen (Rürup, 2007), sind ähnliche Aufmerksamkeitsschwerpunkte in den staatlichen Reformbemühungen zu bemerken (Altrichter \& Heinrich, 2007): Während zunächst unter der Losung von Schulautonomie und erhöhter Eigenverantwortlichkeit von Schulen rechtliche Spielräume sowie Motivation und Energie für Schulentwicklung an den Einzelstandorten geschaffen werden sollte, rückten seit der zweiten Hälfte der 1990er Jahre Fragen der inneren Steuerungsfähigkeit der Einzelschulen (thematisiert durch Reformelemente wie Schulprogramme, Selbstevaluation und Qualitätsmanagement sowie Stärkung der Schulleitung) in den Vordergrund. In dieser Zeit gibt es auch - z.B. unter dem schönen Titel «Die Vielfalt orchestrieren» (EDK, 2000) erste und sanfte administrative Ansätze, die Entwicklungsbemühungen der Schulen in eine bestimmte Richtung zu lenken, z.B. in Form von Aufgabenbeispielen oder durch die Vorgabe, dass Schul- und Entwicklungsprogramme von Einzelschulen auch Entwicklungsschwerpunkte im Bereich «Unterricht» formulieren müssten (Radnitzky, 2002).

In der Folge des PISA-Schocks 2001 erhielten die Bemühungen um die Erneuerung der Steuerungsverhältnisse in den deutschsprachigen Schulsystemen neue Akzente: Die unerwartet ungünstigen Ergebnisse erhöhten den medialen und öffentlichen Druck auf die Bildungspolitik. Diese wollte Handlungsfähigkeit und Reformwillen demonstrieren (Tillmann, Dedering, Kneuper, Kuhlmann \& Nessel, 2008), indem sie deutlicher als zuvor Entwicklungsziele, die in der durch PISA stimulierten Kompetenzsprache abgefasst waren, und energischere 
Eingriffe und Kontrollen der Systemleistung forcierte. Nach und nach kristallisierte sich als Reformziel von Bildungspolitik und Bildungsverwaltung ein Steuerungskonzept heraus, das unter dem Namen «evidenzbasierte Steuerung» den Diskurs von Bildungspolitik und Bildungsreform bestimmt und angesichts des politischen Drucks - relativ rasch zu einem gleichzeitig leistungsfähigeren und ökonomischen Schulsystem führen soll. Was landläufig als «neues Steuerungsmodell» bezeichnet wird, ist dabei nicht unbedingt eine zweifelsfrei umschriebene und homogene Sache (Altrichter \& Maag Merki, 2010, S. 34ff). Dennoch gibt es einige Leitideen, die die Steuerungsreformbemühungen zu einen scheinen. Darunter sind zu zählen:

- Die als notwendig erachtete Umsteuerung braucht einen zentralen und systemweiten Zugriff, um zielgerichtete und schnelle Entwicklungen einzuleiten. Die emphatische und relativ umfassende Autonomie-Losung der beginnenden 1990er Jahre wird zu einer 'Autonomie der (oder mancher) Wege' (Heid, 2003) zurückgenommen.

- Ein wesentlicher Baustein des «evidenzbasierten Steuerungsmodells» besteht darin, die Leistungsziele des Systems klar zu formulieren und den Akteuren im Bildungssystem auch deutlicher als zuvor zu kommunizieren, um damit die Zielorientierung der schulischen Tätigkeit und der Weiterentwicklung von Schule zu verstärken (Klieme, 2004, S. 627). Praktisch sind diese Leistungserwartungen v.a. durch «Bildungsstandards» sowie durch die verschiedenen "Qualitätsrahmen», die den neuen Schulinspektionen unterlegt wurden, signalisiert worden.

- Evaluation und Rechenschaftslegung (accountability) sind weitere Schlüsselthemen dieser Modernisierung der Systemsteuerung. Die Evaluation von Zielen ist systemisch gesprochen auch ein Versuch, die Loyalität der Akteure zu diesen Zielen sicherzustellen. Darüber hinaus hat sie im «evidenzbasierten Steuerungsmodell» noch eine weitere wichtige Funktion: Verschiedene Evaluations- und Systemmonitoringmassnahmen sollen jene "Evidenz» produzieren (zum Begriff vgl. Jornitz, 2008), die erkennen lässt, ob die Leistungserwartungen durch die praktische Tätigkeit der verschiedenen Systemeinheiten erreicht wurden oder nicht.

- Diese «Evidenz» soll in der Folge Systementwicklung stimulieren und in die richtige Richtung weisen. Akteure auf allen Ebenen des Schulsystems - Bildungspolitiker/innen, Verwaltungsbeamte, Schulleitungen, Lehrpersonen, Schüler/innen, Elternvertreter/innen, Schulträger usw. - sollen diese Evaluationsinformationen, die manchmal suggestiv als "Steuerungswissen» bezeichnet werden, dazu verwenden, um rationalere Entwicklungsentscheidungen zu treffen.

- Die Rede von der "Wende in Richtung einer Outputorientierung des Schulsystems» postuliert, dass als Qualitätsindikatoren Informationen über «Leistungen von Bildungseinrichtungen» besonders wichtig wären. Diese werden vor allem im Bereich des fachlichen Unterrichts gesehen und - in 
Weiterentwicklung der PISA-Technologien - über Schülerleistungen geprüft. Daher sind auch für Umsteuerungsbemühungen Veränderungen des Unterrichts ganz entscheidend; ihr letztes Evaluationskriterium finden Systemreformmassnahmen dann in verbesserten Lernergebnissen der Schüler/innen. In diesem Sinne kann man auch von einer Unterrichtsfokussierung der Vorstellungen zum «evidenzbasierten Steuerungsmodell» sprechen.

- Andere Teilelemente des Schulsystems und seiner Umgebung werden als Unterstützungssysteme aufgefasst, die dem operativen Kern - Lernen im Unterricht - und seinen Zielen - repräsentiert durch Bildungsstandards und darauf bezogene Tests - zuarbeiten und von da her ihre Begründung und Existenzberechtigung erhalten.

Diese Leitideen lassen sich zu einem «Regelkreis von Zielbestimmungen, Überprüfungen, Rückmeldungen, Entwicklungsmassnahmen, Unterstützungssystemen und Verbesserungen" zusammensetzen, den Specht (2006, S. 33) als «Idealmodell outputorientierter Steuerung» ansieht. Konkrete Verwirklichungen dieses «Regelkreises» sind die "Standards-Politik» (Maag Merki, 2010) ebenso wie die Einführung von neuen Modellen der «Schulinspektionen» (Husfeldt, 2011; Kotthoff \& Böttcher, 2010). In beiden Fällen werden den Akteuren im Schulsystem Ziele - durch Bildungsstandards und Qualitätsrahmen - vorgegeben; die Erreichung dieser Ziele in der schulischen Arbeit wird - durch Lernstandserhebungen bzw. Teaminspektionen - evaluiert und soll produktive Entwicklungen stimulieren und orientieren.

Wiewohl das «evidenzbasierte Steuerungsmodell» also ein Entwicklungsmodell repräsentiert, das den Impuls zur Systementwicklung v.a. von der Makroebene erwartet, und wiewohl es wichtige Funktionselemente auf verschiedenen Systemebenen und in «Unterstützungssystemen» enthält, scheint - aufgrund der oben erläuterten Interpretation des Prinzips der «Outputorientierung» die Ebene des Unterrichts und des Schülerlernens das zentrale Forum zu sein, auf dem sich letztlich der Erfolg des neuen Steuerungsmodells erweisen wird. Insofern kann die leitende Fragestellung dieses Beitrags auch als zentrale Frage und Erfolgskriterium des «evidenzbasierten Steuerungsmodells» verstanden werden: Wie kann eine «systemweite» Reform im Mehrebenensystem «Schule» betrieben werden, die auch im Unterricht und in den Lernergebnissen der Schüler/innen spürbar wird?

\section{Bildungsstandards, standardbezogene Tests und Datenfeedback als Instrumente systemischer Unterrichtsentwicklung}

Die wichtigste Antwort des «evidenzbasierten Steuerungskonzepts» auf diese Frage lautet gemessen an der Selbstdarstellung der Bildungspolitik und am Umfang ihrer Investitionen (Fend, 2011, S. 7) - Bildungsstandards und standardbezogene Tests. Bildungsstandards sind Zielbeschreibungen von Unter- 
richt und Lernen (und dadurch auch der Schule und des Schulsystems) in einer kompetenzorientierten Sprache. Standards sind aber auch "Instrumente», die etwas bewirken sollen; sie stellen "ein zentrales Gelenkstück» innerhalb «der Anstrengungen zur Sicherung und Steigerung der Qualität schulischer Arbeit dar» (Klieme et al., 2003, S. 9). Standardbezogene Tests, die in den verschiedenen deutschsprachigen Schulsystemen unter verschiedenen Bezeichnungen geläufig sind (wie z.B. Lernstandserhebungen), sollen die Schülerleistungen in Hinblick auf diese Ziele erheben und damit jene "Evidenzen", die als wesentliche Indikatoren für die Leistungsfähigkeit des Systems angesehen werden. Diese Informationen sollen den wesentlichen Akteuren zurückgefüttert werden, um dadurch Bemühungen der weiteren Qualitätsentwicklung zu stimulieren und $\mathrm{zu}$ orientieren. "Jedenfalls wurde der Erhebung und Rückmeldung von Lernergebnissen eine zentrale Funktion bei der Steuerung (...) des Schulsystems beigemessen, die wiederum Grundlage für die Weiterentwicklung der Bildungsqualität sei» (Klieme, 2004, S. 626).

Die verschiedenen Instrumente evidenzbasierter Steuerung haben eine Monitoringaufgabe für das Gesamtsystem»; sie sollen die Informationsbasis für die Entscheidungsorgane auf höheren Systemebenen verbessern. Aber nicht nur. Sie haben auch einen Verbesserungsanspruch, der letztlich auch am selbst propagierten Kriterium der Schülerleistung zu bemessen ist. Und sie wollen ihre Entwicklungsimpulse nicht nur an die Ebene der Makrosteuerung von Politik und hoher Verwaltung senden, sondern auch an die Ebenen von Schulleitungen, Lehrpersonen und Schüler/innen, indem sie aufwändige Systeme des Datenfeedbacks konstruieren, die die operativen Akteure mit Evidenzen für die Weiterentwicklung versorgen sollen.

\section{Wie können Bildungsstandards "steuern"?}

Mit dem heute so aktuellen Begriff «Steuern» bezeichnet man in sozialen Zusammenhängen einen einigermassen komplexen Sachverhalt: «Steuern» meint, (1) dass soziale Akteure Handlungen setzen, die andere soziale Akteure stimulieren, auf ihrer jeweiligen Handlungsebene weitere Handlungen zu setzen, die ihrerseits weitere Akteure zu Handlungen ... usw. ad libitum. Und (2) ist damit mitgedacht, dass dieses Geflecht von Handlungen und Folgehandlungen letztlich das Gesamtsystem in Richtung eines angestrebten Zielbereiches weiterentwickelt. Im Falle von sozialen Entwicklungen wird man Ziele nicht zu eng beschreiben, sondern eher im Sinne von akzeptablen Zielkorridoren. Dennoch: wer steuern will, kann nicht mit jedem möglichen Ergebnis zufrieden sein.

In einem Mehrebenensystem - wie dem Schulwesen - kann man nicht erwarten, dass die rechtliche Fassung und administrative Verordnung von Neuerungen schon allein zu einer Veränderung der Tätigkeit des Schulsystems und seiner Ergebnisse führen. Neuerungen müssen auf den verschiedenen Ebenen aufgegriffen und verstanden werden; und sie müssen - wie Fend (2006b, S. 181) dies sehr «sprechend» bezeichnet hat - für den Kontext der jeweiligen 
Ebene «rekontextualisiert» werden, d.h. in - dieser Ebene entsprechende Handlungen und Arbeitsstrukturen übersetzt werden: Schulen müssen sich z.B. fragen, ob ihre Leitideen und praktischen Unterrichtsprogramme den geforderten Kompetenzzielen entsprechen. Lehrpersonen müssen Bildungsstandards zu zentralen Orientierungspunkten ihrer Planungen, ihrer Unterrichtsgestaltung und ihrer Leistungsbeurteilung machen. Schüler/innen müssten richtig liegen, wenn sie aktive und eigenverantwortliche Lernprozesse an diesen Leitsternen orientieren, während regionale Verwaltung und Supportsysteme ihre Vorgaben und Unterstützungsangebote diesen Zielbeschreibungen gemäss gestalten.

\section{Können Bildungsstandards "steuern"?}

Was dürfen wir aufgrund bisheriger Forschungserfahrung erwarten: Werden Bildungsstandards die erhoffte «Steuerungs»» Wirkung entfalten können? Wenn wir die heute aktuelle Steuerungssprache kurz verlassen und Bildungsstandards aus einer pädagogischen Perspektive betrachten, dann sind diese konkreten kompetenzorientierten Zielbeschreibungen eigentlich curriculare Elemente, die sich weder inhaltlich noch formal grundlegend von früheren unterrichtsbezogenen Lernzielbeschreibungen in den Lehrplänen unterscheiden (Fend, 2008, S. 295). Aus der Lehrplanforschung (Höhmann, 2002; Vollstädt, Tillmann \& Rauin, 1999; Wiater, 2006) wissen wir, dass Lehrpläne und mit ihnen die curricularen Zielbeschreibungen - freundlich ausgedrückt - äusserst träge Steuerungsinstrumente sind, die jedenfalls kaum das Instrument der Wahl für eine Bildungsreform wären, durch die eine Weiterentwicklung des Unterrichts in einem absehbaren Zeitraum betrieben werden sollte.

Warum sollten Bildungsstandards Unterricht besser "steuern» als die Zielbeschreibungen in Lehrplänen? Weil sie durch Aufgabenbeispiele konkretisiert werden? Weil die Lehrpersonen in besonderer Weise bereit und qualifiziert sind, diese Neuerung aufzugreifen? Weil ihre Umsetzung durch besondere Implementierungsmassnahmen unterstützt wird? Weil die Beziehung zwischen Standards und Tests einen besonderen Druck in Richtung ihrer Nutzung ausüben wird? Fend (2008, S. 292) scheint der letzteren Interpretation zuzuneigen, wenn er vorhersagt, dass Bildungsstandards erst «im Verbund mit einer entfalteten Testpraxis zu einem Paradigmenwechsel von der ,Systemsteuerung' hin zu einer neuen ,Governance'» führen werden.

\section{Lehrpersonen und Bildungsstandards}

In den deutschsprachigen Schulsystemen sind in den letzten Jahren zum Teil gewaltige Anstrengungen (Fend, 2011, S. 7) unternommen worden, Bildungsstandards auszuarbeiten und darauf bezogene Testsysteme ins Laufen zu bringen. Welche Erfahrungen hat man damit gemacht? Hat die Formulierung und Propagierung von Bildungsstandards die Arbeit im Schulsystem in einer solchen Weise und in einem solchen Umfang verändert, dass man tatsächlich von einer «neuen Steuerung», die bis zur Ebene der Unterrichts- und Lernhandlungen 
geht, sprechen kann? Eine abschliessende Antwort auf diese Frage kann sicher noch nicht gegeben werden: Die Fachleute sind sich auch uneinig, mit welchen Zeiträumen man von der Verordnung von Bildungsstandards über deren Rezeption und «Implementierung» in den Schulen bis zu den ersten spürbaren Wirkungen in Schülerlernen und -leistungen rechnen müsse. Was aber heute wohl schon gesagt werden kann, ist, dass sich die meisten dieser Fachleute den Weg bis zur schulischen Rezeption, Nutzung und Wirkung von Bildungsstandards leichter vorgestellt haben.

Nehmen wir als Beispiel Österreich. Das hat den Vorteil, dass man die dort erhobenen Daten nicht als gültig für die verschiedenen deutschsprachigen Schulsysteme ansehen muss, sie aber - aufgrund der ähnlicher Schulstruktur und Reformschwerpunkte - als Frageheurismen für die Analyse des eigenen Systems verwenden kann. In Österreich wurden seit den ersten Pilotversuchen mit der Implementierung von Bildungsstandards immer wieder Befragungen von Lehrpersonen und Schulleiter/innen durchgeführt, die analysieren sollten, "wie die Bildungsstandards und die damit im Zusammenhang stehenden Implementierungsmassnahmen von den Lehrerinnen/Lehrern und Schulleiterinnen/ Schulleitern rezipiert werden» (Grillitsch, 2010, S. 98).

Vorab ein paar Worte zur spezifischen Bedeutung der neuen Steuerungsinstrumente im österreichischen Kontext: Bildungsstandards sind als sogenannte «Regelstandards» konzipiert. Sie legen jene Kompetenzen fest, die Schüler/innen bis zum Ende der 4. Schulstufe in Deutsch/Lesen/Schreiben und in Mathematik sowie bis zum Ende der 8. Schulstufe in Deutsch, Lebende Fremd-sprache/ Englisch und Mathematik «in der Regel» bzw. im «Durchschnitt» nachhaltig erworben haben sollen (Grillitsch, 2010, S. 5). Ein Unterstützungssystem, das aus Informations-, Begleit- und Fortbildungsmassnahmen für Multiplikator/ innen, Führungskräfte und Lehrende sowie aus Publikationen unterrichtsnaher Lehr-, Lern- und Begleitmaterialien besteht, soll die dauerhafte Verankerung der Bildungsstandards im Unterricht ermöglichen (vgl. www.bifie.at/bildungsstandards).

Parallel zur Ausarbeitung der Standards wurden standardbezogene Tests als Instrumente zur Überprüfung der Standard-Kompetenzen entwickelt. Die Rückmeldung der Resultate dieser Tests erfolgt via Internet. Dazu erhalten Schüler/innen, Lehrer/innen sowie die Schulleitung jeweils Zugangscodes. Die Schüler/innen können ausschliesslich ihr individuelles Ergebnis einsehen, Lehrer/innen erhalten die aggregierten Ergebnisse ihrer Klasse (nicht aber die individuellen Schülerleistungen) und die Schulleitung erhält das aggregierte Gesamtergebnis der Schule. Durch diese Art der Rückmeldung soll der Kontrolldruck auf den jeweiligen Ebenen vermindert werden (Grabensberger, Freudenthaler \& Specht, 2008, S. 13). Als Teil des Unterstützungssystems innerhalb des Rückmeldeprozesses kommen in Österreich speziell ausgebildete «Rückmeldemoderatorinnen und -moderatoren» zum Einsatz, die die Interpretation und Nutzung der Evaluationsergebnisse durch die Betroffenen unterstützen und bei 
der Konzeption und Umsetzung von unterrichtsbezogenen Entwicklungsmassnahmen beratend zur Seite stehen sollen (Grabensberger et al., 2008, S. 15f.).

Bei den im Folgenden diskutierten Studien handelt es sich jeweils um Befragungen von Schulleiter/innen, deren Schulen in den jeweiligen Jahren an der Pilotierung (bzw. bei Grillitsch (2010) an der baseline-Testung) der Bildungsstandards teilgenommen hatten, bzw. aller von der Pilotierung betroffenen Lehrkräfte dieser Schulen. Die Befragungen wurden mit standardisierten Fragebogen vom staatlichen Bundesinstitut für Bildungsforschung, Innovation und Entwicklung des österreichischen Bildungswesens (BIFIE) bzw. dessen Vorgängerinstitutionen durchgeführt und veröffentlicht. Alle Daten und, soweit möglich, für empirische Untersuchungen übliche Kennwerte wurden diesen Publikationen entnommen. Eine kritische Diskussion der bis dahin erschienenen Untersuchungen haben wir an anderer Stelle vorgelegt (Altrichter, 2008, S. 80ff.). Die Daten der unterschiedlichen Jahre sind aufgrund der wechselnden Grösse der Stichproben, unterschiedlicher Rücklaufquoten und leicht variierender Fragestellungen nicht in allen Einzelheiten vergleichbar, doch lassen sich u.E. mit der gebotenen Vorsicht einige Aussagen und kritische Fragen ableiten:

(1) Etwa die Hälfte der befragten Lehrpersonen, die in den Pilotprojekten Bildungsstandards kennen gelernt hatten, sehen durchaus hilfreiche Nutzungsmöglichkeiten von Standards für Unterrichtsplanung, Lernstandsdiagnose und Selbstreflexion der Lehrkräfte (Tab. 1). Allerdings nimmt die Zahl der Zweifler im Verlauf der beobachteten fünf Jahre kaum ab - mit Ausnahme der sehr bedeutsamen Nutzung für die Unterrichtsplanung.

Tab. 1: Wie hilfreich sind Bildungsstandards für ...? Antworten österreichischer Lehrpersonen verschiedener Stichproben (Freudenthaler \& Specht, 2005, S. 42, 2006, S. 15f:; Grillitsch, 2010, S. 77)

$\begin{array}{lcccc} & \begin{array}{c}\text { Lehrkräfte von } \\ \text { Sekundarschulen } \\ 2004 ; \mathrm{n}=115\end{array} & \begin{array}{c}\text { HS- Lehrkräfte } \\ 2005 ; \\ \mathrm{n}=468\end{array} & \begin{array}{c}\text { AHS- Lehrkräfte } \\ 2005 ; \\ \mathrm{n}=292\end{array} & \begin{array}{c}\text { Lehrkräfte von } \\ \text { Sekundarschulen } \\ 2009 ; \mathrm{n}=1270\end{array} \\ \begin{array}{l}\text { hilfreich für Diagnose des } \\ \begin{array}{l}\text { Lernstandes } \\ \text { hilfreich für Planung und }\end{array}\end{array} & 65 \% & 63 \% & 47 \% & 60 \% \\ \begin{array}{l}\text { Gestaltung eines kompeten- } \\ \text { zorientierten Unterrichts }\end{array} & 42 \% & 57 \% & 45 \% & 52 \% \\ \begin{array}{l}\text { hilfreich für Selbstreflexion } \\ \text { der Lehrkraft }\end{array} & 51 \% & 1) & 1) & 53 \%\end{array}$

Legende: HS = Hauptschule; AHS = Gymnasiale Langform

1) Diese Antwortalternative ist in Freudenthaler \& Specht (2006, S. 16) nicht dokumentiert...;

2004 und 2005 wurden als Antwortmöglichkeiten «ja» und «nein» vorgegeben; die angegebenen Prozentwerte beziehen sich auf die Antwortalternative «ja».

2009 enthielt die Skala 7 Antwortmöglichkeiten von «sehr hilfreich» bis «gar nicht hilfreich». Die angegebenen Prozentwerte beziehen sich jeweils auf die Summe der zustimmenden Antwortalternativen 1 - 3 . 
(2) Trotz dieser Einschätzung möglicher nützlicher Anwendungen ist die Zahl jener, die Bildungsstandards zusätzlichen Orientierungswert gegenüber Lehrplänen zubilligen, deutlich geringer und steigt auch nicht zwischen den beiden Beobachtungszeitpunkten (Tab. 2).

Tab 2:: Bringen Ihrer Ansicht nach die Standards gegenüber den Lehrplänen zusätzliche Klärung und Orientierung für die Unterrichtsarbeit?2 Antworten österreichischer Lehr- und Schulleitungspersonen verschiedener Stichproben (Freudenthaler \& Specht, 2005, S. 50; Grillitsch, 2010, S. 81)

Lehrkräfte von Sekundarschulen 2004; $n=115$

ja, eindeutig

kaum

weiss ich noch nicht

nein

eher Verwirrung
Lehrkräfte von Sekundarschulen 2009; $\mathrm{n}=1270$

$16 \%$

$29 \%$

$33 \%$

$13 \%$

$9 \%$
Schulleiter/innen von Sekundarschulen 2009; $\mathrm{n}=185$

$36 \%$

$27 \%$

$28 \%$

$4 \%$

$5 \%$

1) wurde 2004 nicht als Antwortalternative vorgegeben.

(3) Die Gruppe jener Lehrpersonen, die den potentiellen Orientierungswert von Bildungsstandards auch tatsächlich konsequent für die eigene Unterrichtsplanung nutzt, ist noch kleiner und scheint im Verlaufe der Beobachtungszeit noch zu schrumpfen (Tab. 3). Die etwas grösser werdende Gruppe jener, die angibt, sie versuche es «hin und wieder», ist schwer einzuschätzen.

Tab. 3: Verwenden Sie die Standards für Ihre Unterrichtsplanung bzw. -gestaltung?3 Antworten österreichischer Lehrpersonen verschiedener Stichproben (Freudenthaler \& Specht, 2005, S. 31, 2006, S. 17; Grillitsch, 2010, S. 91f.)

\begin{tabular}{|c|c|c|c|c|c|}
\hline & $\begin{array}{c}\text { Lehrkräfte } \\
\text { von Sekundar- } \\
\text { schulen 2004; } \\
\mathrm{n}=115\end{array}$ & $\begin{array}{l}\text { VS- Lehrkräfte } \\
2005 ; \\
n=82\end{array}$ & $\begin{array}{l}\text { HS- Lehrkräfte } \\
\begin{array}{c}2005 ; \\
n=468\end{array}\end{array}$ & $\begin{array}{l}\text { AHS- } \\
\text { Lehrkräfte } \\
\text { 2005; } \\
\mathrm{n}=292\end{array}$ & $\begin{array}{c}\text { Lehrkräfte } \\
\text { von Sekundar- } \\
\text { schulen } 2009 \\
n=1270\end{array}$ \\
\hline $\begin{array}{l}\text { ja, regelmässig } \\
\text { [04: ja, intensiv und } \\
\text { regelmässig }\end{array}$ & $14 \%$ & $11 \%$ & $2 \%$ & $2 \%$ & $7 \%$ \\
\hline $\begin{array}{l}\text { hin und wieder [04: } \\
\text { ich versuche es hin } \\
\text { und wieder] }\end{array}$ & $56 \%$ & $58 \%$ & $51 \%$ & $41 \%$ & $62 \%$ \\
\hline $\begin{array}{l}\text { nein } \\
\text { [04: nie] }\end{array}$ & $30 \%$ & $31 \%$ & $47 \%$ & 58 \% & $31 \%$ \\
\hline
\end{tabular}

Legende: VS = Grundschule, HS = Hauptschule; $A H S$ = Gymnasiale Langform 
(4) Dazu passt, dass die Nützlichkeit von Bildungsstandards für die eigene Unterrichtsarbeit eher skeptisch eingeschätzt wird; ganz wenige Lehrpersonen erwarten sich eine Erleichterung ihrer Tätigkeit (Tab. 4).

\section{Tab. 4: Stellt die Arbeit mit Standards eher eine Erleichterung oder eine} Erschwernis der Unterrichtsarbeit dar?4 Antworten österreichischer Lehr- und Schulleitungspersonen verschiedener Stichproben (Freudenthaler \& Specht, 2005, S. 51, 2006, S. 24; Grillitsch, 2010, S. 85)

\begin{tabular}{|c|c|c|c|c|c|}
\hline & $\begin{array}{l}\text { Lehrkräfte } \\
\text { von Sekundar- } \\
\text { schulen '04; } \\
\text { n=115 }\end{array}$ & $\begin{array}{l}\text { HS-Lehrkräfte } \\
\begin{array}{c}2005 ; \\
n=468\end{array}\end{array}$ & $\begin{array}{c}\text { AHS- } \\
\text { Lehrkräfte } \\
2005 ; \\
n=292\end{array}$ & $\begin{array}{l}\text { Lehrkräfte } \\
\text { von Sekundar- } \\
\text { schulen '09; } \\
\text { n=1270 }\end{array}$ & $\begin{array}{c}\text { Schulleiter/ } \\
\text { innen von } \\
\text { Sekundar- } \\
\text { schulen'09; } \\
\mathrm{n}=185\end{array}$ \\
\hline eher Erleichterung & $15 \%$ & & & $10 \%$ & $10 \%$ \\
\hline weder noch & $60 \%$ & & & $46 \%$ & $41 \%$ \\
\hline eher Erschwernis & $25 \%$ & & & $21 \%$ & $21 \%$ \\
\hline $\begin{array}{l}\text { weiss ich noch } \\
\text { nicht }\end{array}$ & 1) & & & $23 \%$ & $28 \%$ \\
\hline $\begin{array}{l}\text { Nutzen grösser als } \\
\text { Aufwand }\end{array}$ & & $22 \%$ & $17 \%$ & & \\
\hline
\end{tabular}

1) wurde 2004 nicht als Antwortalternative vorgegeben.

(5) Beim Vergleich unterschiedlicher Lehrergruppen zeigt sich, dass der Informationsstand, das Ausmass der erlebten Unterstützung und die Teilnahme an standardbezogenen Fortbildungsveranstaltungen mit einer positiveren Einschätzung von Bildungsstandards, einer höheren Nutzeneinschätzung und einer häufigeren Verwendung für Unterrichtsplanung zusammenhängt (Grillitsch, 2010, S. 99f.). Dies alles lässt sich so interpretieren, dass Unterstützung der Lehrpersonen und Schulen bei der Nutzung von Bildungsstandards im Unterricht ein gewisses Potenzial hat; die in den Tabellen mitgeteilten Zahlen im Zeitverlauf deuten an, dass dieses Potenzial noch weit davon entfernt ist, gehoben zu werden.

(6) Zusätzliche Fallstudien (Aiglsdorfer \& Aigner, 2005; Hölzl \& Rixinger, 2007) deuten schliesslich darauf hin, dass «Bildungsstandards» bei den Schülerinnen und Schülern in nur sehr eingeschränkter Bedeutung angekommen sind: In den dabei untersuchten Pilotschulen assoziierten Schüler/innen den Begriff vor allem mit Tests sowie mit Aufgabenbeispielen im Unterricht; sie hatten aber nicht gelernt, diese Kompetenzbeschreibungen für eigenverantwortliche Lernplanung und Lerngestaltung zu nutzen.

«Sind Bildungsstandards ein wirksames Instrument zur Steuerung der Weiterentwicklung von Unterricht?» Die bisherigen Erfahrungen scheinen darauf hinzudeuten, dass bis zur systemischen Wirksamkeit noch ein weiter Weg ist. Wie bei anderen Innovationen auch, kommt Veränderung nicht durch 
ein gutes Konzept und seine administrative Verordnung, sondern durch das Aufgreifen und Weiterentwickeln solcher Ideen an verschiedenen Stellen des Mehrebenensystems, v.a. aber auf Schul- und Unterrichtsebene: Die Reform gewinnt Gestalt in der «Implementation». Die letztere allerdings scheint schwerer zu sein als erwartet. Bildungsstandards können offenbar kaum von sich aus Unterrichtsentwicklung stimulieren, sondern benötigen viel eher Unterrichtsentwicklung als Voraussetzung und Begleitung, benötigen entwickelte Fähigkeiten für kompetenzorientiertes Unterrichten, um «wirksam» zu werden.

Die massive Zunahme an Fortbildungsveranstaltungen und Publikationen zum Thema «kompetenzorientierter Unterricht» zeigt, dass dieser Sachverhalt nun erkannt ist. Auch neue Lehrpläne nehmen expliziter auf «Kompetenzorientierung» Bezug (als Beispiel die Hamburger Bildungspläne). Viel weniger findet sich zur Frage, wie Bildungsstandards an Schüler/innen herangebracht werden können, sodass sie von diesen in produktiver Weise für ihr Lernen genutzt werden können.

\section{Lernstandserhebungen und Datenfeedback}

Wie steht es nun mit dem zweiten Element der «evidenzbasierten Steuerung», von dem man sich dynamisierenden und orientierenden Einfluss auf die Weiterentwicklung des Unterrichts erwartet: mit dem Feedback der Ergebnisse standardbezogener Tests, die Unterrichtsentwicklung anregen und in die richtige Richtung leiten sollen.

In Tab. 5 finden sich weitere Daten aus der österreichischen Begleituntersuchung, diesmal nach der ersten Rückmeldung der Ergebnisse der standardbezogenen Tests erhoben. Die Interpretation der Evaluator/innen Grabensberger et al. (2008, S. 71) lautet folgendermassen: "Die Wirkungen, die die Ergebnisrückmeldungen zu den Standard-Testungen auf die Schule als Organisation gezeitigt haben - beispielsweise in der Form von gemeinsam getragenen Einsichten, Beschlüssen oder Schulentwicklungsmassnahmen - halten sich in engen Grenzen. Zwar bekunden etwa $30 \%$ der Befragten, dass die Kommunikation im Kollegium über pädagogische Fragen durch die Tests und ihre Ergebnisse intensiviert worden sei, und $22 \%$ meinen, dass man sich heute an der Schule mehr mit Fragen der Schul- und Unterrichtsqualität beschäftige. Dies hat aber nur in sehr wenigen Fällen dazu geführt, dass etwa konkrete Beschlüsse oder Massnahmen getroffen worden sind. Die Aussage mit der höchsten Zustimmungsrate bei den Lehrkräften ist, es habe sich, wenig verändert' ( $-60 \%)$. Dies gilt allerdings auch in Bezug auf mögliche problematische Aspekte: Konflikte, Spannungen, Schuldzuweisungen oder Ähnliches als Folge von Problemrückmeldungen werden von jeweils weniger als $10 \%$ der Lehrpersonen berichtet.» 
Tab. 5: Hat die Standardtestung bzw. Ergebnisrückmeldung an Ihrer Schule zu konkreten Reaktionen geführt? Antworten österreichischer Lehrpersonen (n=487; Grabensberger et al., 2008, S. 71f.)

\begin{tabular}{|l|c|c|c|c|c|}
\hline Aussagen & $\begin{array}{c}\text { Zu- } \\
\text { stimmung } \\
(1-2)\end{array}$ & $\begin{array}{c}\text { Neutral } \\
(3)\end{array}$ & $\begin{array}{c}\text { Ab- } \\
\text { lehnung } \\
(4-5)\end{array}$ & M & SD \\
\hline $\begin{array}{l}\text { Die Kommunikation im Kollegium über } \\
\text { pädagogische Fragen wurde angeregt }\end{array}$ & $29 \%$ & $34 \%$ & $38 \%$ & 3.21 & 1.03 \\
\hline $\begin{array}{l}\text { Die Lehrkräfte an meiner Schule } \\
\text { beschäftigen sich heute intensiver mit } \\
\text { Themen der Schul- und Unterrichts- } \\
\text { qualität }\end{array}$ & $22 \%$ & $42 \%$ & $36 \%$ & 3.26 & 0.95 \\
\hline $\begin{array}{l}\text { Lehrkräfte bemühen sich heute stärker } \\
\text { um einen besseren Unterricht }\end{array}$ & $17 \%$ & $38 \%$ & $45 \%$ & 3.49 & 1.06 \\
\hline $\begin{array}{l}\text { In der Gesamtkonferenz wurden die } \\
\text { Ergebnisse der Standardtestung disku- } \\
\text { tiert }\end{array}$ & $18 \%$ & $19 \%$ & $63 \%$ & 3.84 & 1.23 \\
\hline $\begin{array}{l}\text { Es sind verbindliche Beschlüsse im } \\
\text { Hinblick auf Veränderungsmaßnahmen } \\
\text { getroffen worden }\end{array}$ & $5 \%$ & $14 \%$ & $81 \%$ & 4.29 & 0.91 \\
\hline $\begin{array}{l}\text { Es sind vermehrt Konflikte und } \\
\text { Spannungen innerhalb des Kollegiums } \\
\text { aufgetreten }\end{array}$ & $9 \%$ & $14 \%$ & $78 \%$ & 4.21 & 1.04 \\
\hline $\begin{array}{l}\text { Lehrkräfte mit schlechteren und } \\
\text { solche mit besseren Ergebnissen haben } \\
\text { sich konstruktiv ausgetauscht und } \\
\text { gemeinsam nach Lösungen gesucht }\end{array}$ & $6 \%$ & $22 \%$ & $72 \%$ & 4.06 & 0.94 \\
\hline $\begin{array}{l}\text { Lehrkräfte haben verstärkt Kritik an } \\
\text { Rahmenbedingungen geäußert }\end{array}$ & $50 \%$ & $25 \%$ & $25 \%$ & 2.66 & 1.31 \\
\hline $\begin{array}{l}\text { Eigentlich hat sich nicht viel verändert } \\
\text { (1) }\end{array}$ & $61 \%$ & $27 \%$ & $12 \%$ & 2.25 & 1.05 \\
\hline
\end{tabular}

Legende: Antworten auf 5-stufigen Skalen von (1) trifft völlig zu bis (5) trifft überhaupt nicht zu

Wiederum könnte es sich bei diesen Daten um «Ausreisser» handeln oder um die Widerspiegelung einer «zu kurzen Wirkungsdauer» einer Intervention. Ein Überblick über bisherige Studien, in denen die Auswirkungen von Datenfeedback auf Schul- und Unterrichtsentwicklung untersucht wurden (Altrichter, 2010a), zeigt in seiner Zusammenfassung: In den bisherigen Implementationsversuchen in den deutschsprachigen Schulsystemen hat Datenfeedback enttäuschend wenig konsistente Entwicklung nach sich gezogen. Wir haben überhaupt nur eine Studie (Tresch, 2007) gefunden, aus der man Hoffnung 
und sachliche Hinweise für Weiterentwicklung schöpfen könnte.

Diese Ergebnisse waren auch insofern überraschend, als viele Kritiker/innen der Bildungsstandards-Politik sogar «übersteuernde» Wirkungen befürchtet hatten: Die Vorgabe und Prüfung von Standards würden - wie in den angelsächsischen Ländern vielfach beobachtet (Koretz, 2008; Maag Merki, 2010; Nichols \& Berliner, 2007) - zu "Teaching to the Test», zu einer Verengung des Curriculums und zur Ungleichbehandlung unterschiedlicher Schülergruppen führen. Die Ergebnisse deutschsprachiger Studien mögen in manchen Fällen mit suboptimalen Umsetzungsversionen dieser Neuerung zusammenhängen. Die bisherigen Untersuchungen stützen sich zudem beinahe ausschliesslich auf Selbstberichte der Lehrkräfte und vernachlässigen potentielle Einflussfaktoren (Altrichter, 2010a, S. 253f.). Am plausibelsten erscheint jedoch, dass der bildungspolitische Kontext einen Unterschied macht: Die high stakes-Struktur der anglo-amerikanischen Bildungssysteme produziert bedeutend mehr Rezeptions- und Umsteuerungsdruck als die meist traditionell administrativ geprägte Implementation der neuen Instrumente in den deutschsprachigen Bildungssystemen.

Insgesamt entsteht jedenfalls der Eindruck, dass häufig überzogene Erwartungen an das Instrument Datenfeedback und sein Potenzial zur Rationalisierung und Steuerung der Entwicklung von Schule und Unterricht im Kontext der deutschsprachigen Schulsysteme vorliegen (van Ackeren, 2007, S. 207). Datenfeedback ist als Steuerungsinstrument ausgesprochen voraussetzungsvoll. Rückgemeldete Daten über Prozesse, Bedingungen und Ergebnisse von Unterricht und Schule «steuern» nicht direkt, sondern können eine etwaige Steuerungswirkung nur über vielfache Vermittlungsprozesse über verschiedene Ebenen des Mehrebenensystems «Schule» hinweg entfalten - Vermittlungsprozesse, die sich aus rezeptiven und konstruktiven Aktivitäten verschiedener Akteure auf der Basis bestehender Einstellungen und Kompetenzen und in bestimmten strukturellen Bedingungen zusammensetzen. Feedbackdaten sind ein Element in einem Steuerungskontext, in dem von Entscheidungsakteuren noch weitere Informationen und andere Rücksichten beachtet werden müssen. In der Realität eines Mehrebenensystems ist Evaluation «ein unspezifischer Impuls für die sukzessive Veränderung von Programmen, nicht ein zielsicher umsetzbarer Plan für eine ad hoc zu vollziehende Umgestaltung» (Kuper, 2005, S. 101f.). Entwicklung aufgrund von Evaluation geschieht «eher im Sinne eines allmählichen, von aussen kaum steuerbaren Einsickerns, als im Sinne einer punktgenauen Implementation von Informationen» (Kuper, 2005, S. 103). Feedbackinformationen im diskutierten Sinn sind ein «Beitrag zur Unterstützung der Verhandlung zwischen den Akteuren bzw. Akteursgruppen» (Kuper, 2005, S. 101) und nicht eine Entschärfung der Ambiguität von individuellen und organisationellen Entscheidungssituationen oder ein Ersatz (mikround makro-)politischer Aushandlungsprozesse zwischen Akteuren mit divergierenden Interessen. 
Welche Konsequenzen für Bildungspolitik und Schulreform ergeben sich aus der vorliegenden Analyse? Die allgemeinen Schlüsse sind recht klar: Dämpfung der überzogenen Erwartungen, realistische Reformulierung der angestrebten Funktionen. Wenn man aus Gründen gesellschaftlicher Transparenz und einer engeren Orientierung der Schulen an Systemzielen eine stärkere Beachtung solcher Informationen für Entwicklungsentscheidungen in Unterricht und Schule will, dann braucht es

- intensivere Unterstützungsleistungen und gezieltere Implementationsbemühungen,

- die auch differentielle Angebote für unterschiedliche Bedarfsgruppen enthalten

- und sich auf einen längerfristigen und schrittweisen Prozess der Veränderung von Einstellungen und Praktiken sowie von Kultur- und Strukturmerkmalen in Einzelschulen und Schulsystem einrichten.

Dazu braucht es aber auch eine Klärung in Hinblick auf die widersprüchlichen Signale, die von testbasierten Leistungsrückmeldungen ausgehen, die da sind (O’Day, 2004):

- die Leistung einzelner Schüler/innen wird gemessen, einzelne Lehrer/innen und/oder die Schule als Einheit sollen handeln;

- Messungen, die nicht selten als externe Kontrollmechanismen verstanden werden, sollen interne Operationen auslösen;

- Output-Messungen sollen prozessbezogen interpretiert werden und Aktivitäten zur Entwicklung des Unterrichtsprozesses nach sich ziehen;

- eine Politik, die aus einem Zweifel an der professionellen Selbstentwicklungsfähigkeit der Lehrpersonen und Schulen entstanden ist, appelliert an die Professionalität des schulischen Personals, aus Datenrückmeldung Konsequenzen der Qualitätsentwicklung zu ziehen.

Gerade im letzten Punkt scheint das Grunddilemma der evidenzbasierten Bildungspolitik zu liegen: Wenn die Nutzung von Lernstandserhebungen einen (kompetenzorientierten) «Paradigmenwechsel» im Lehrerhandeln, wenn sie eine «neue Professionalität» erfordert, dann setzt die Steuerungsinstrumente Bildungsstandards und Datenfeedback gerade etwas voraus, zu dem sie selbst beitragen sollten und dessen Mangel als Ausgangspunkt für die neueren Entwicklung diagnostiziert wurde.

\section{Unterrichtsreform durch Fortbildung und Entwicklungsprogramme}

Wir kommen zur zweiten Fragerichtung: Gibt es erfolgreiche Strategien der Unterrichtsentwicklung, die ein Potenzial für systemweite Reform haben? Wenn man in Deutschland nach relativ breiten und erfolgreichen Programmen der Unterrichtsentwicklung fragt, so bekommt man meist «SINUS»5 (Prenzel, 
Carstensen, Senkbeil, Ostermeier \& Seidel, 2005) als Antwort, vielleicht noch zusätzlich das Projekt "Chemie im Kontext (ChiK) ${ }^{6}$ " (Parchmann \& Gräsel, 2006). In Österreich wird die Antwort «IMST»7 (Krainer, 2007) und «PFL $8_{»}$ (Altrichter \& Posch, 1998) lauten. Die Tatsache, dass vielen Gewährspersonen einige wenige Programme als Antwort einfallen, interpretieren wir so:

- Es gibt gar nicht so viele Programme der Unterrichtsentwicklung, die über die Propagierung von Einzelmassnahmen hinausgehen und einen breiten Anspruch - sowohl in Hinblick auf die Zahl der erfassten Personen als auch auf die Reichweite der didaktischen Innovationen - haben.

- Solche Programme erreichen eine gewisse Publizität in der Szene.

- Nachfolgeprogramme noch weiter gehenden Anspruchs scheinen selten zu sein.

Wir können diese Programme hier nicht im Einzelnen darstellen, doch scheinen sie mindestens folgende gemeinsame Elemente aufzuweisen:

- Längerfristigkeit: Zunächst handelt es sich dabei nicht um kurzzeitige Fortbildungsangebote des Typs «ein Thema - ein/e Referent/in - ein Halb-/Ganz-/ Doppeltag», sondern um «Programme», die sich zwar verschiedener Formen der Fortbildung bedienen, aber darüber hinaus noch weitere «organisatorische» Elemente aufweisen und länger dauern.

- Ausgehen von praktischen Problemsichten und wissenschaftlich fundierte Impulse für die Unterrichtsentwicklung: Einesteils greifen diese Programme üblicherweise Problemlagen und Problemsichten der Praxis auf. Andererseits versuchen sie, theoriegeleitete Impulse durch pädagogische und fachdidaktische Konzepte zu geben und oft auch entsprechende Unterstützungsmaterialien anzubieten. So bietet SINUS Lernmodule zu didaktischen Themen an, die als Problembereiche des mathematisch-naturwissenschaftlichen Unterrichts identifiziert wurden (Prenzel et al., 2005, S. 541f.).

- Unterrichtsentwicklung als zentrale Lerntätigkeit und Aufgabe: Die Weiterentwicklung des eigenen Unterrichts ist in diesen Programmen keine Aufgabe, die nach der Fortbildung in Angriff genommen wird, sondern wesentliches Element der Fortbildung selbst: Impulse aus Projektrahmen und Unterstützungsmaterialien sowie aus den kooperativen Lehrertreffen sollen für den Unterricht weitergedacht und umgesetzt, die damit gemachten Erfahrungen ausgewertet und für die weitere Entwicklung der Lehrerkompetenzen und des Unterrichts genutzt werden.

- Aktiv-entwickelnde und reflektierende Haltung der Lehrpersonen gegenüber eigenem Unterricht: Das didaktische und organisatorische Arrangement dieser Programme soll Lehrpersonen stimulieren, eine Haltung und Praxis aktiven und selbstbestimmenden Lernens und der kritischen Reflexion eigener Tätigkeit zu entwickeln. So werden in "ChiK» innerhalb des Projektrahmens verschiedene Arbeitsmöglichkeiten angeboten; die letztlich bearbeiteten Arbeitsschwerpunkte werden von den Lehrpersonen ausgewählt. «... zur Umsetzung der gemeinsamen Vorhaben wurden Unterrichtseinheiten und -materialien entwickelt, im 
Unterricht erprobt und in weiteren gemeinsamen Treffen - insgesamt 6-8 pro Schuljahr - reflektiert und überarbeitet.» (Parchmann \& Gräsel, 2006, S. 157) In den österreichischen Programme IMST und PFL finden sich verschiedene Arrangements, die Lehrkräfte anregen sollen, fokussierte Entwicklungsarbeit im eigenen Unterricht zu betreiben, deren Verlauf, Bedingungen und Ergebnisse zu reflektieren und anderen Lehrpersonen in «kollegialer Lehrerfortbildung» zur Diskussion zu stellen (Altrichter \& Posch, 1998). SINUS will Schulen zur Zielüberprüfung mit einfachen Verfahren der Selbstreflexion anregen (Prenzel et al., 2005, S. 542).

- Lehrerkooperation: Durch Arrangements der Lehrerkooperation - bei «ChiK» «Lerngemeinschaften», bei SINUS «Sets», bei PFL «Regionalgruppen» genannt - sollen kollegiale Impulse für Entwicklung und Reflexion bereitgestellt sowie die Motivation für eine kontinuierliche Weiterentwicklung unterstützt werden.

- Perspektive auf die Backhome-Situation: Alle genannten Programme arbeiten nicht mit ganzen Schulen oder (Fach-)Kollegien, sondern - hier typisch im Fortbildungsformat - mit einzelnen Lehrpersonen, wobei die Tendenz, zumindest Paare oder kleine Teams aus der gleichen Schule gewinnen zu wollen, feststellbar ist. Diesem - innovationstheoretischen - Mangel versuchen die Programme oft dadurch entgegenzuarbeiten, indem sie die backhome-Situation thematisieren und zum Gegenstand von Lernen und Übung machen, z.B. indem für die Arbeit in der Fachgruppe vorbereitet wird oder Kompetenzen der kollegialen Fortbildung geübt werden.

Alle diese Programme sind auch begleitend evaluiert worden. Obgleich eine Wirkungsevaluation von Lehrerfortbildung aufgrund der vielfältigen Einflüsse jenseits der Fortbildung, denen Lehrpersonen in langfristigen, praxiseinbeziehenden Programmen unterliegen, und der langen «Wirkungsketten» von der Lehrereinstellung und -kognition über das Unterrichtshandeln bis zu den Schülererfahrungen (Hattie, 2009, S. 120; Lipowsky, 2010, S. 52ff.) eine Reihe von Fallen bietet, werden die Evaluationsergebnisse im Allgemeinen als ermutigend interpretiert, wenngleich sie nicht ohne Widersprüche und Nachdenkimpulse sind. So zeigen sich bei SINUS konzeptentsprechende Ergebnisse über die Kette von Lehrerwahrnehmung, Schülerwahrnehmung bis zu Lernergebnissen von Schülern, jedoch nicht in allen Schulformen in gleicher Weise (Prenzel et al., 2005). Parchmann und Gräsel (2006, S. 158) konstatieren für "Chemie im Kontext» grosse Lehrerakzeptanz und konzeptentsprechende Erfolge, aber auch eine "grosse Heterogenität in der Wahrnehmung und in gezeigten Lernerfolgen.» Für die österreichischen PFL-Lehrgänge wird berichtet, dass sie Lehrpersonen, die an fachdidaktischen Themen und an der persönlichen Kompetenzentwicklung interessiert sind, anziehen. Dennoch steigen im Verlauf der Lehrgänge Kompetenz in der Reflexion eigener Praxis, selbstberichtete Unterrichtskompetenz, die Beteiligung an Schulentwicklungsprozessen sowie lehrgangsspezifische Wissensbereiche weiter an. Die Ergebnisse 
bei einer auf Video präsentierten Anwendungsaufgabe zeigen, «dass dem aktiven Tun, dem Anwenden und Üben eine hohe Bedeutung in der Lehrerfortbildung zukommt", allerdings auch, dass Transfer der Inhalte der Fortbildung eher bei solchen Aufgaben gelingt, die nahe den im Lehrgang geübten Arbeitsinhalten und -formen waren (Müller, Andreitz \& Mayr, 2010, S. 198).

Die genannten Programme scheinen jedenfalls eine Reihe jener Merkmale ertragreicher Lehrerfortbildung in praktikable und für die Teilnehmer/innen attraktive didaktische und organisatorische Arrangements zu kleiden, die beispielsweise Lipowsky $(2004,2010)$ ähnliche Hinweise geben Bessoth (2007), Messner und Reusser (2000), Richardson und Placier (2001) aus einer Zusammenschau internationaler Befunde der Lehrerbildungsforschung herausgearbeitet hat:

- Qualität des Lernprozesses

o längerfristige Auseinandersetzung mit Inhalten

o Vielfalt methodischer Settings

o kritisches Hinterfragen grundlegender Überzeugungen

- Bezug zum Unterricht und zur eigenen Handlungspraxis

o klarer Bezug zur Unterrichtspraxis der Teilnehmer/innen

o fachdidaktischer Fokus auf ausgewählte Fragestellungen

- Strukturelle Einbettung und Stimulierung systemkoordinativer Beziehungen

o Anregung zur Kooperation, die über die Veranstaltung hinausgeht

o Teilnahme mehrerer Lehrkräfte eines Standortes

o externe Unterstützung bei Umsetzung der Fortbildungsinhalte an der Schule

Entscheidende Faktoren eines Lehrerlernens, das Chancen zur Weiterentwicklung eigenen Unterrichts bietet, scheinen einerseits in ganz naheliegende Merkmalen zu liegen: Produktive Lehrerfortbildung sieht das Lernen von Lehrer/innen als eine ernsthafte, längerfristige, aktive und kommunikative Auseinandersetzung mit eigener Handlungspraxis und den zugrunde liegenden Wissens- und Einstellungselementen an. Sie findet in einem Bezug zur eigenen Handlungspraxis statt; Distanzierung von bestehender Praxis geschieht hier nicht durch die Themen oder die Tagungsorte der Fortbildung, sondern als «reflexive Distanzierung» (Hameyer, 1984, S. 175) in der entwickelnden und evaluierenden Auseinandersetzung mit eigener Praxis. Andererseits scheinen Faktoren wichtig zu sein, die das Lernen der Lehrpersonen über die Fortbildung hinaus mit einem Netz sozialer Beziehungen und sachlicher Entwicklungsaufgaben in ihrer Arbeitssituation verbinden können.

\section{Zusammenfassende Diskussion}

Wie kann eine «systemweite» Reform im Mehrebenensystem «Schule» betrieben werden, die auch im Unterricht und in den Lernergebnissen der Schüler/innen 
spürbar wird? lautete die Ausgangsfrage. Zusammenfassend lässt sich unserer Einschätzung nach festhalten:

- Der Anspruch der verschiedenen Varianten des sogenannten «evidenzbasierten Steuerungsmodells» und seiner Kernelemente Bildungsstandards, standardbezogene Tests und Datenrückmeldung liegt in einer Veränderung von Unterricht, Schülerlernen und dessen Ergebnisse.

- Die "wirkungsvolle Implementation» dieser Vorstellung stellte sich als schwieriger als erwartet heraus, lag doch die Hoffnung auf einer schnelleren und effektiveren «Umsteuerung» (als durch langwierigere «schulautonome» Entwicklungsprozesse) in einer bildungspolitisch unbefriedigenden und druckvollen Situation.

Bisher wurde bei der Implementation von Bildungsstandards vor allem auf den technischen Ebenen der Standardsformulierung und Testentwicklung gearbeitet. Die erforderlichen Aktivitäten auf der Schul-und Unterrichtsebene werden vielerorts noch unterschätzt und kaum gesehen. Sie müssen dringend ebenfalls in einer langfristigen Perspektive verstärkt werden, wenn das zu etablierende Testsystem nicht wirkungslos bleiben oder sogar kontraproduktiv werden soll (Oelkers \& Reusser, 2008, S. 509).

- In der Lehrerbildungsforschung liegt durchaus schon einiges Wissen über wichtige Merkmale von Fortbildung vor, die Lehrpersonen zur Weiterentwicklung ihres Unterrichts anregt und sie dabei unterstützt. Und es scheint auch Programme zu geben, die diese Merkmale in einer praktikablen Weise zu organisieren und umzusetzen vermögen. Unter den üblichen Bedingungen des Schulsystems erreichen diese Programme allerdings oft nur spezielle Gruppen von Lehrpersonen, die tendenziell günstigere Einstellungen zu aktivem Lernen und der Weiterentwicklung ihres Unterrichts aufweisen (Müller et al., 2010, S. 198).

- Die genannten Programme scheinen ihr Potenzial für Unterrichtsentwicklung gerade aus einer Intensität und Kontinuität des Arbeitens abzuleiten, die sich üblicherweise eher in Teilbereichen, selten aber aus Personal- und Ressourcengründen - auf "ganzer Breite» des Systems herstellen lassen. Einer der Autor/ innen (HA) kann sich noch lebhaft erinnern, dass beim ersten Lesen der Studie über die Implementation von Bildungsstandards von Oelkers und Reusser (2008, S. 417ff.) Enttäuschung angesichts der dort vorgeschlagenen "Werkzeuge und Verfahren» der Lehrerfortbildung aufkam. Sowohl das dort vorgeschlagene «fachspezifisch-pädagogische Coaching (Staub, 2001) als auch die "videobasierte Analyse und Reflexion von Unterricht» (Reusser, 2005) können sich in Hinblick auf Aufwand und Langfristigkeit der Lernprozesse durchaus mit den hier kommentierten Fortbildungsprogrammen messen? Nach und nach wurde mir bewusst, dass die Vorschläge von Oelkers und Reusser (2008) wohl nicht als Hinweise für eine «schnelle Re-Education auf ganzer Breite» taugen, wohl aber als ernsthafter Hinweis darauf zu werten sind, dass Lernen und Umlernen langfristige und intensive Lernkontexte 
benötigen. Dass diese «erheblicher Investitionen und der «Bündelung und Umschichtung von Ressourcen» bedürfen (Reusser, persönl. Mitteilung, 21.5.2007), ist dann nur konsequent.

Das Szenario, das Oelkers und Reusser (2008) aufbauen, ist also nach unserer Einschätzung ebenso zutreffend wie frustrierend. Es ist realpolitisch frustrierend, weil man sich mit Recht fragen wird, ob nach dem finanziellen und emotionalen Engagement der Bildungspolitik für "Neue Steuerung» tatsächlich noch Kraft für aufwändige, die Akteure ernsthaft mit einbeziehende Unterrichtsentwicklung vorhanden ist. Es ist aber wahrscheinlich auch in Hinblick auf die bildungspolitische Prioritätensetzung der vergangenen Jahre frustrierend, weil die neuen Steuerungsinstrumente Bildungsstandards und Datenfeedback ja gerade Versuche waren, sich vom Wollen und Meinen der Lehrpersonen unabhängiger zu machen und «rationaleren», systemeinheitlichen Steuerungsparametern mehr Gewicht zu verleihen. Was hier und bei Oelkers und Reusser (2008) als zukunftsträchtige Modelle für Unterrichtsentwicklung identifiziert wird, verlangt aber wieder die - «aufwändige» - Auseinandersetzung mit den existierenden Lehrerrationalitäten.

- Eine «Unterrichtsreform» mit dem Anspruch, in spürbarer Systembreite Schülerlernen und dessen Ergebnisse zu verändern, erfordert einen komplexen sozialen Lernvorgang, in dem eine «strukturelle Reform durch eine (lern-)kulturelle Reform begleitet» (Oelkers \& Reusser, 2008, S. 266) wird. Oelkers und Reusser (2008) haben die dabei auftauchenden Ansprüche ausführlich beschrieben. Wir wollen für diese Zusammenfassung nur mehr einige Gesichtspunkte hervorheben (Altrichter \& Feyerer, 2012).

- Eine solche Reform braucht einesteils die Entwicklung von einigermassen kohärenten Strukturen, die Entwicklungsarbeit und Lernen im Sinne der Zielsetzungen fordern und fördern. Dies geht von den Schulgesetzen und -verordnungen über Lehrmaterialproduktion und systemtypische "Incentives» zu den «Unterstützungssystemen» in Ausbildung und Fortbildung. Das Ziel muss «ein ,alignment' von Strukturen, Strategien, Regelungen und Materialien im Sinne der Zielsetzung der Reform und dies auf allen Ebenen des Systems» (Oelkers \& Reusser, 2008, S. 257) sein.

- Gerade weil eine gewisse «Kohärenz» der systemischen Signale für eine «Umsteuerung» notwendig ist, diese aber nicht zu einem totalitären pädagogischen System ausarten dürfen, müssen auch die Spielräume, die den einzelnen Akteuren, Schulen und Regionen eröffnet werden, klarer als bisher gemacht werden. Der obligatorische Verweis auf «Schulautonomie» genügt hier nicht, weil sich das, was «autonome Spielräume» vor Ort sein können, seit den 1990er Jahren deutlich gewandelt hat und in einer «evidenzbasierten Steuerung» neu definiert werden muss.

- Eine solche Reform muss eben auch die Akzeptanz und das Engagement der Akteure vor Ort, vor allem der Lehrpersonen, Schulleitungen und - noch immer unterschätzt - der Schüler/innen gewinnen, die sich auf (professio- 
nelles) Lernen, Unterrichts- und Schulentwicklung einlassen müssen. Und sie muss sie in Bildungs- und Entwicklungsprozesse involvieren, die ihnen sowohl attraktiv erscheinen, als sie auch zur Weiterentwicklung von Unterricht und Lernen herausfordern und sie dabei unterstützen.

- Kaskadenartigen Verbreitungsmodellen, die hier oft als Ausweg, der sowohl Intensität als Breite gewährleisten soll, gewählt werden und in denen Programmteilnehmer/innen ihre Erfahrungen back home am Arbeitsort weitergeben sollen, fällt es nach den Erfahrungen der Innovationsforschung oft schwer, die Intensität der Originale auf den unteren Stufen der Kaskade aufrecht zu erhalten. Die Evaluation von SINUS, in der «flächendeckende Effekte auf Schulebene» (Prenzel et al., 2005, S. 559), nicht nur solche bei speziellen SINUS-Lehrkräften festgestellt wurden, legt jedoch nahe, den Transfer an der Heimat-Schule der Teilnehmer/innen ernst zu nehmen und als spezielles Lern- und Unterstützungsfeld im Entwicklungsprogramm selbst zu bearbeiten.

- «Unterrichtsreform» ist aktuell - wahrscheinlich wie die meisten herausfordernden Aufgaben - eine sehr widersprüchliche Angelegenheit: Sie soll individuelle und soziale Akteure stimulieren, ihre Ressourcen für Ziele einzusetzen, die andere entwickelt haben und die sie sich ? im günstigsten Fall zum Teil zu eigen machen. Zentrale politische und administrative Akteure veranstalten sie, weil sie keine Hoffnung haben, dass die autonomen Selbstentwicklungskräfte der verschiedenen Systemakteure in gleicher Geschwindigkeit zu den gleichen Zielen führen würden; andererseits sind sie auf das Engagement und die Rationalität gerade dieser Akteure, deren Handeln sie beschleunigen und orientieren wollen, angewiesen, um ihre Ziele zu erreichen.

\section{Amerkungen}

1 Eine ähnliche Argumentation liesse sich für das Reformelement «Schulinspektion» aufbauen; da jedoch «Bildungsstandards und Datenfeedback» ihrem Anspruch nach deutlich «unterrichtsfokussierter» sind, erscheinen sie für unser Thema «Unterrichtsentwicklung» geeigneter.

22004 enthielt die Fragestellung nicht die Wendung «für die Unterrichtsarbeit» (Freudenthaler \& Specht 2005, S. 50).

3 In der Untersuchung 2004 lautete die Frage «Verwenden Sie derzeit die Standards für Ihre laufende Unterrichtsplanung? " (Freudenthaler \& Specht, 2005, S. 31). In der Untersuchung 2005 lautete die Frage: «Haben Sie die Standards - abgesehen von der Erprobung von Beispielaufgaben - für die Unterrichtsplanung im laufenden Schuljahr verwendet?» (Freudenthaler \& Specht, 2006, S. 17). Die spezifizierte Fragestellung könnte eine Erklärung für den extrem niedrigen Wert sein.

42004 enthielt die Fragestellung nicht die Wendung "der Unterrichtsarbeit» (Freudenthaler \& Specht, 2005, S. 51). 2005 lautete die Frage: «Wenn Sie den Arbeitsaufwand, den die Erprobung der Standards mit sich gebracht hat, dem daraus resultierenden Nutzen gegenüber stellen, was ergibt sich daraus für Sie?» (Freudenthaler \& Specht, 2006, S. 24).

5 Das BLK-Programm SINUS («Steigerung der Effizienz des mathematisch-naturwissenschaftlichen Unterrichts») hatte es sich zum Ziel gesetzt "Prozesse der Qualitäts- 
sicherung und Optimierung von Lehren und Lernen in den mathematisch-naturwissenschaftlichen Fächern in Gang zu setzen und zu unterstützen» (Gutzer, 2005, S. 33). Eine zentrale Rolle spielte dabei die Professionalisierung der Lehrkräfte und die Qualitätsverbesserung des Unterrichts mit Hilfe von Modulen (Prenzel et al., 2005, S. 542).

6 «Chemie im Kontext» (ChiK) war ein theoriegeleitetes Projekt der Unterrichtsentwicklung, das verschiedenen Problemen des Chemieunterrichts, wie nachlassendem Interesse, ungünstige Einstellungen gegenüber dem Fach und ungenügendem Konzeptverständnis entgegen arbeiten sollte (Parchmann \& Gräsel, 2006, S. 156).

7 IMST («Innovationen machen Schulen top») ist ein Programm der Universität Klagenfurt, mit dem der Unterricht in Mathematik, Naturwissenschaften, Informatik und Deutsch verbessert werden soll. Ziel ist die Förderung anspruchsvoller Fähigkeiten und mehr Selbständigkeit bei den Schüler/innen sowie die professionelle Weiterentwicklung der Lehrer/ innen durch Erfahrungsaustausch und Kooperation (Krainer, 2008, S. 345).

8 Das zweijährige Lehrerfortbildungsprogramm «Pädagogik und Fachdidaktik für Lehrer/ innen» wurde Ende der 1980er Jahre an der Universität Klagenfurt entwickelt und sollte durch eine aktiv-forscherische Haltung gegenüber eigenem Unterricht fachdidaktische Entwicklung von Lehrpersonen anregen (Altrichter, 2010b; Altrichter \& Posch, 1998).

9 Und mit anderen vielversprechenden aktuellen Entwicklungen, wie z.B. den «lesson studies» (Fernandez, 2002; Kullmann, 2011; White \& Southwell, 2003)

\section{Literatur}

Ackeren, I. van (2007). Nutzung grossflächiger Tests für die Schulentwicklung: Exemplarische Analyse der Erfahrungen aus England, Frankreich und den Niederlanden. Bildungsforschung (Bd. 3). Bonn: Bundesministerium für Bildung und Forschung.

Aiglsdorfer, B. \& Aigner, M. (2005). Implementierung nationaler Bildungsstandards in Österreich. Unveröffentlichte Diplomarbeit, Johannes Kepler Universität, Linz.

Altrichter, H. (2008). Veränderungen der Systemsteuerung im Schulwesen durch die Implementation einer Politik der Bildungsstandards. In T. Brüsemeister \& K.-D. Eubel (Hrsg.), Evaluation, Wissen und Nichtwissen (S. 75-115). Wiesbaden: Verlag für Sozialwissenschaften.

Altrichter, H. (2010a). Schul- und Unterrichtsentwicklung durch Datenrückmeldung. In H. Altrichter \& K. Maag Merki (Hrsg.), Handbuch Neue Steuerung im Schulsystem (S. 219-254). Wiesbaden: Verlag für Sozialwissenschaften.

Altrichter, H. (2010b). Lehrerfortbildung im Kontext von Veränderungen im Schulwesen. In F. H. Müller, A. Eichenberger, M. Lüders \& J. Mayr (Hrsg.), Lehrerinnen und Lehrer lernen (S. 17-34). Münster: Waxmann.

Altrichter, H. \& Feyerer, E. (2012). Auf dem Weg zu einem inklusiven Schulsystem? Zeitschrift für Inklusion, O (4). Zugriff am 08.02.2012 unter http://www.inklusion-online. net/index.php/inklusion/article/view/131/127

Altrichter, H. \& Heinrich, M. (2007). Kategorien der Governance-Analyse und Transformationen der Systemsteuerung in Österreich. In H. Altrichter, T. Brüsemeister \& J. Wissinger (Hrsg.), Educational Governance - Handlungskoordination und Steuerung im Bildungssystem (S. 55-103). Wiesbaden: VS.

Altrichter, H. \& Maag Merki, K. (Hrsg.). (2010). Handbuch Neue Steuerung im Schulsystem. Wiesbaden: VS.

Altrichter, H. \& Posch, P. (1998). Einige Orientierungspunkte für 'nachhaltige Lehrerfortbildung'. In H.-J. Herber \& F. Hofmann (Hrsg.), Schulpädagogik und Lehrerbildung (S. 245-259). Innsbruck: Studien Verlag.

Bessoth, R. (2007). Wirksame Weiterbildung. Oberentfelden: Sauerländer.

Brüsemeister, T. \& Eubel, K.-D. (Hrsg.). (2003). Zur Modernisierung der Schule. Bielefeld: Transcript. 
EDK. (2000). Die Vielfalt orchestrieren. Innsbruck: Studien Verlag.

Fend, H. (2006a). Geschichte des Bildungswesens. Wiesbaden: VS.

Fend, H. (2006b). Neue Theorie der Schule. Wiesbaden: VS.

Fend, H. (2008). Die Bedeutung von Bildungsstandards im Kontext von Educational Governance. Beiträge zur Lehrerbildung, 26, 292-303.

Fend, H. (2011). Die Wirksamkeit der Neuen Steuerung - theoretische und methodische Probleme ihrer Evaluation. Zeitschrift für Bildungsforschung, 1, 5-24.

Fernandez, C. (2002). Learning from Japaneses approaches to professional development: The case of lesson study. Journal of Teacher Education, 53, 393-405.

Freudenthaler, H. H. \& Specht, W. (2005). Bildungsstandards aus Sicht der Anwender. ZSE-Report Nr. 69. Graz: ZSE.

Freudenthaler, H. H. \& Specht, W. (2006). Bildungsstandards: Der Implementationsprozess aus der Sicht der Praxis. Graz: ZSE.

Grabensberger, E., Freudenthaler, H. H. \& Specht, W. (2008). Bildungsstandards: Testungen und Ergebnisrückmeldungen auf der achten Schulstufe aus der Sicht der Praxis. Graz: bifie.

Grillitsch, M. (2010). Bildungsstandards auf dem Weg in die Praxis. BIFIE-Report 6/2010. Graz: Leykam.

Gutzer, H. (2005). Das Sinus-Transfer-Modell für Schulen. Schulverwaltung, 15 (1), 33-35.

Hameyer, U. (1984). Interventive Erziehungsforschung. In H. Haft \& H. Kordes (Hrsg.), Methoden der Erziehungs- und Bildungsforschung (S. 145-181). Stuttgart: Klett-Cotta.

Hattie, J. (2009). Visible learning. Abingdon: Routledge.

Heid, H. (2003). Standardsetzung. Zeitschrift für Pädagogik, 47. Beiheft, 176-193.

Höhmann, K. (2002). Was wird durch eine Lehrplanrevision verändert? Frankfurt am Main: Lang.

Hölzl, L. \& Rixinger, G. (2007). Implementierung von Bildungsstandards in Österreich - das zweite Jahr. Unveröffentlichte Diplomarbeit, Johannes Kepler Universität, Linz.

Husfeldt, V. (2011). Wirkungen und Wirksamkeit der externen Schulevaluation. Überblick zum Stand der Forschung. Zeitschrift für Erziehungswissenschaft, 14 (2), 259-282.

Jornitz, S. (2008). Was bedeutet «evidenzbasierte Bildungsforschung»?Die Deutsche Schule, $100,206-216$.

Klieme, E. (2004). Begründung, Implementation und Wirkungen von Bildungsstandards: Aktuelle Diskussionslinien und empirische Befunde. Zeitschrift für Pädagogik, 50, 625-634.

Klieme, E., Avenarius, H., Blum, W., Döbrich, P., Gruber, H., Prenzel, M., Reiss, K., Riquarts, K., Rost, J., Tenorth, H.-E. \& Vollmer, H. J. (2003). Zur Entwicklung nationaler Bildungsstandards. Eine Expertise. Bonn: Bundesministerium für Bildung und Forschung.

Koretz, D. (2008). Test-based educational accountability. Research evidence and implications. Zeitschrift für Pädagogik, 54, 777-790.

Kotthoff, H.-G. \& Böttcher, W. (2010). Neue Formen der «Schulinspektion». In H. Altrichter \& K. Maag Merki (Hrsg.), Handbuch neue Steuerung im Schulwesen (S. 295-325). Wiesbaden: VS.

Krainer, K. (2007). Die Programme IMST und SINUS: Reflexionen über Ansatz, Wirkungen und Weiterentwicklungen. In D. Höttecke (Hrsg.), Naturwissenschaftlicher Unterricht im internationalen Vergleich (S. 20-48). Berlin: Lit.

Krainer, K. (2008). Genese, Ansatz und Wirkungen des Projekts IMST. In F. Hofmann, C. Schreiner \& J. Thonhauser (Hrsg.), Qualitative und quantitative Aspekte (S. 343-357). Münster: Waxmann.

Kullmann, H. (2011). Lesson study - Eine konsequente Form unterrichtsbezogener Lehrerkooperation. Unveröffentlichtes Manuskript, Universität Bielefeld.

Kuper, H. (2005). Evaluation im Bildungssystem. Stuttgart: Kohlhammer.

Lipowsky, F. (2004). Was macht Fortbildungen für Lehrkräfte erfolgreich? Die deutsche Schule, 4, 462-479. 
Lipowsky, F. (2010). Lernen im Beruf. Empirische Befunde zur Wirksamkeit von Lehrerfortbildung. In F. H. Müller, A. Eichenberger, M. Lüders \& J. Mayr (Hrsg.), Lehrerinnen und Lehrer lernen (S. 51-70). Münster: Waxmann.

Maag Merki, K. (2010). Theoretische und empirische Analysen der Effektivität von Bildungsstandards, standardbezogenen Lernstandserhebungen und zentralen Abschlussprüfungen. In H. Altrichter \& K. Maag Merki (Hrsg.), Handbuch Neue Steuerung im Schulsystem (S. 145-169). Wiesbaden: VS.

Messner, H. \& Reusser, K. (2000). Berufliches Lernen als lebenslanger Prozess. Beiträge zur Lehrerbildung, 18 (3), 277-294.

Müller, F. H., Andreitz, I. \& Mayr, J. (2010). PFL — Pädagogik und Fachdidaktik für Lehrerinnen und Lehrer. Eine Studie zu Wirkungen forschenden Lernens. In F. H. Müller, A. Eichenberger, M. Lüders \& J. Mayr (Hrsg.), Lehrerinnen und Lehrer lernen (S. 183-202). Münster: Waxmann.

Nichols, S. L. \& Berliner, D. C. (2007). Collateral damage. How high-stakes testing corrupts America's schools. Cambridge: Harvard Education Press.

O'Day, J. A. (2004). Complexity, accountability, and school improvement. In S. H. Fuhrman \& R. F. Elmore (Eds.), Redesigning accountability systems for education (pp. 15-43). New York: Teachers College Press.

Oelkers, J. \& Reusser, K. (2008). Qualität entwickeln - Standards sichern - mit Differenz umgehen. Berlin: Bundesministerium für Bildung und Forschung.

Parchmann, I. \& Gräsel, C. (2006). Chemie im Kontext - Wege der Verbreitung innovativer Ansätze in der Schule. In A. Pitton (Hrsg.), Lehren und Lernen mit neuen Medien (S. 156-158). Münster: LIT.

Prenzel, M., Carstensen, C. H., Senkbeil, M., Ostermeier, C. \& Seidel, T. (2005). Wie schneiden SINUS-Schulen bei Pisa ab? Zeitschrift für Erziehungswissenschaften, 8, 540-562.

Radnitzky, E. (2002). Q.I.S. - Schulprogrammkonzept und Service-Angebot des Bildungsministeriums. In F. Eder \& H. Altrichter (Hrsg.), Qualitätsentwicklung und Qualitätssicherung im österreichischen Schulwesen (S. 153-168). Innsbruck: StudienVerlag.

Reusser, K. (2005). Situiertes Lernen mit Unterrichtsvideos in der Lehrerinnen- und Lehrerbildung. Journal für Lehrerinnen- und Lehrerbildung 5 (2), 8-18.

Richardson, V. \& Placier, P. (2001). Teacher change. In V. Richardson (Ed.), Handbook of research on teaching (pp. 905-947). Washington, DC: AERA.

Rürup, M. (2007). Innovationswege im deutschen Bildungssystem. Wiesbaden: VS.

Specht, W. (2006). Von den Mühen der Ebene. Entwicklung und Implementation von Bildungsstandards in Österreich. In F. Eder, A. Gastager \& F. Hofmann (Hrsg.), Qualität durch Standards? (S. 13-37). Münster: Waxmann.

Staub, F. C. (2001). Fachspezifisch-pädagogisches Coaching: Förderung von Unterrichtsexpertise durch Unterrichtsentwicklung. Beiträge zur Lehrerbildung, 19, 175-198.

Tillmann, K.-J., Dedering, K., Kneuper, D., Kuhlmann, C. \& Nessel, I. (2008). PISA als bildungspolitisches Ereignis. Wiesbaden: VS.

Tresch, S. (2007). Potenzial Leistungstest. Wie Lehrerinnen und Lehrer Ergebnisrückmeldungen zur Sicherung und Steigerung ihrer Unterrichtsqualität nutzen. Bern: h.e.p.

Vollstädt, W., Tillmann, K.-J. \& Rauin, U. (1999). Lehrpläne im Schulalltag. Opladen: Leske+Budrich.

White, A. L. \& Southwell, B. (2003). Lesson study project: Evaluation report. State of NSW. Department of Education and Training. Zugriff am 03.05.2011 unter http://www. curriculumsupport.education.nsw.gov.au/secondary/mathematics/assets/pdf/lesson_stdy/ lesson_study_eval_03.pdf

Wiater, W. (2006). Lehrplan, Curriculum, Bildungsstandards. In K.-H. Arnold, U. Sandfuchs \& J. Wiechmann (Hrsg.), Handbuch Unterricht (S. 169-178). Bad Heilbrunn: Klinkhardt. 
Schlagworte: Unterrichtsentwicklung, neues Steuerungsmodell, Bildungsstandards, evidenzbasierte Steuerung, Lehrerfortbildung, Governance

\section{Évolution des cours au moment de la réforme du système}

\section{Ré sumé}

Depuis la première moitié des années 1990, on peut constater, dans les différents systèmes scolaires de langue allemande, des mouvements renforcés de changements qui, dans leur ensemble, ont été interprétés comme constituant des tentatives de réforme de la gestion scolaire. Ceci étant, l'innovation concernant les cours est placée de manière renforcée en point de mire des exigences de réforme depuis la seconde moitié des années 1990. C'est dans cet esprit que le contributeur se pose la question de savoir comment une réforme peut être menée pour tout le système dans sa globalité dans le système à plusieurs niveaux constitué par l'école, sachant qu'elle sera ressentie dans les cours et les résultats des apprentissages pédagogiques des écolières et des écoliers. A ce sujet, on y fournit dans un premier temps quelques explications en vue de nous permettre de comprendre le «nouveau pilotage» et la place qui y est donné aux cours (voir la section 1), pour ensuite analyser les effets des instruments de réforme actuellement en place «Standards de formation, tests par rapport aux standards et au feedback des données» sur le pilotage et le développement des cours (voir la section 2). Dans la section 3 - dans le cadre d'un revirement de l'orientation de la question - on analyse finalement des stratégies d'évolution des cours ayant connu un succès quant à leur potentiel pour des réformes couvrant l'ensemble du système.

Mots-clés: Evolution des cours, nouveau modèle de pilotage, standards de formation, pilotage basé sur les évidences, formation continue des maîtres, Governance.

\section{Lo sviluppo in aula ai tempi della riforma del sistema}

\section{Riassunto}

Fin dalla prima metà degli anni '90, nei diversi sistemi scolastici nei Paesi di lingua tedesca, si è notato un aumento dei movimenti di riforma, che sono stati interpretati complessivamente come un tentativo di modificare l'apparato scolastico. In questo senso, l'innovazione dell'insegnamento, dalla seconda metà degli anni '90, sposta prepotentemente il focus sulle proposte di modifica. In questo senso, l'articolo si pone la questione, su come si possa attuare una riforma in un sistema scolastico multilivello, che è percepibile agli scolari / alle scolare anche per ciò che riguarda l'insegnamento e i risultati dell'apprendimento. A questo proposito, vengono in seguito fornite alcune chiarificazioni per la nostra 
comprensione riguardo al «nuovo apparato» e all'importanza dell'insegnamento (si veda Cap. 1), per analizzare successivamente l'effetto degli strumenti di riforma odierni e attuali «Standard formativi, test basati su standard e feedback dei dati» sull'apparato e sull'uteriore sviluppo dell'insegnamento (si veda Cap. 2). Infine, nel Capitolo 3 si discute - in un' inversione di direzione della questione delle strategie di successo dello sviluppo dell'insegnamento e del loro potenziale nell'ambito delle riforme del sistema.

Parole chiave: Sviluppo dell'insegnamento, nuovo modello dell'apparato, standard formativi, apparato basato sull'evidenza, sviluppo professionale degli insegnanti, governance

\title{
Classroom Development in Times of System Reform
}

\begin{abstract}
Since the first half of the 1990s school systems in German speaking countries have seen a growing stream of political debates and initiatives attempting to transform the governance mode of the education system. Within these discourses the call for innovating teaching and learning has become increasingly important. The paper discusses the question how a system-wide educational reform may be pursued which makes a difference for classroom practice and student learning. Firstly, we explain our understanding of the present policies of «new governance» and the significance of «classroom development» within this concept. Secondly, we discuss some data with respect to the effects of «new «overnance» instruments (such as performance standards, standard-related tests and data feedback) on the regulation and development of classroom practice. In chapter 3 we look at successful strategies of classroom development and discuss their potential for system-wide reform.
\end{abstract}

Key words: Classroom development, new models of governance, performance standards, evidence-based governance, professional development of teachers 
